Pacific Journal of Mathematic 


\section{A REPRESENTATION THEOREM FOR MEASURES ON INFINITE DIMENSIONAL SPACES}

\section{Franz Harpain and Maurice Sion}

If $X$ is a locally compact, regular topological space, then the well known Riesz representation theorem sets up an isomorphism between the family of all bounded Radón outer measures on $X$ and the set of continuous positive linear functionals on the family of continuous functions with compact support in $X$. In this isomorphism corresponding elements, $l$ a linear functional and $\mu$ a measure, satisfy the relationship $l(f)=\int f d \mu$ for all continuous functions $f$ with compact support in $X$.

Since an infinite product of locally compact, regular spaces is in general no longer locally compact with respect to the product topology, the Riesz representation theorem fails to hold for such spaces. In this paper, an analogue of the Riesz representation theorem is obtained for this case.

The main idea is to replace the various families mentioned above by the following:

(i) A family $\mathscr{C}$ of cylinders whose elements act like compact sets for a "pseudo-topology" $\mathscr{G}$, where $\mathscr{G}$ is closed under finite intersections and countable unions and is a subset of the product topology.

(ii) A family $M$ of bounded outer measures, related to $\mathscr{C}$ and $\mathscr{G}$ in much the same way as bounded Radón outer measures are related to compact and open sets.

(iii) A family $F$ of functions depending only on a finite number of coordinates, with respect to which they are continuous and have compact support.

(iv) A family $L$ of positive linear functionals on the linear span of $F$.

Under the added hypothesis of $\sigma$-compactness of the coordinate spaces, we show that $L$ and $M$ are isomorphic in such a way that corresponding elements, $l$ in $L$ and $\mu$ in $M$, satisfy the relationship $l(f)=\int f d \mu$ for all $f$ in $F$.

Moreover we show that the elements of $\boldsymbol{M}$ can be viewed as the projective limit measures of projective systems of bounded regular Borel measures.

From the integrability of the members of $F$, it follows that all bounded Borel functions which depend only on a finite number of coordinates are also integrable. Thus the simple functions used by Šilov [7] and the tame functions used by Segal [6] and Gross [2] in the development of an 
integration theory on Hilbert space are included among the integrable functions of the measures considered here. (For a good guide to the literature in this area see the bibliography in Gross [3].) Our results therefore not only characterize an important class of linear functionals in terms of projective limits of regular Borel measures, but also enable one to extend these functionals to a much wider class of functions through a standard integral with respect to a measure, thereby obviating the need to develop a special theory of integration in infinite dimensional spaces for this purpose.

\section{General notation.}

(1) $\varnothing$ is the empty set.

(2) $\omega$ is the set of natural numbers.

(3) $\boldsymbol{R}$ is the set of real numbers.

(4) $\mathscr{C}$ is a compact family if and only if for every subfamily $\mathscr{A}$ of $\mathscr{C}$, if the intersection of any finite number of members of $\mathscr{A}$ is nonvoid, then the intersection of all members of $\mathscr{A}$ is nonvoid.

(5) For $f$ a function on $X$ to $\boldsymbol{R}$ and $A \subset X$,

$f \mid A$ is the restriction of $f$ to $A$,

$1_{A}$ is the characteristic function of $A$,

$\|f\|_{\infty}=\sup \{|f(x)|: x \in X\}$,

$f^{+}(x)=\max \{0, f(x)\}$ for $x \in X$,

support $f=$ closure $\{x: f(x)>0\}$ if $X$ is a topological space.

(6) If for $n \in \omega, \alpha_{n}$ is a set, $a_{n} \in \boldsymbol{R}, f_{n}$ is a function on $X$ to $R$, then $\alpha_{n} \uparrow \alpha$ if and only if $\alpha_{n} \subset \alpha_{n+1}$ and $\mathrm{U}_{n \in \omega} \alpha_{n}=\alpha$,

$a_{n} \uparrow a$ if and only if $a_{n} \leqq a_{n+1}$ and $\lim _{n \in \omega} a_{n}=a$,

$f_{n} \uparrow f$ if and only if for all $x \in X, f_{n}(x) \leqq f_{n+1}(x)$ and $\lim _{n \in \omega} f_{n}(x)=f(x)$.

(7) For $I$ an index set and $X_{i}$ a set for each $i \in I$, $\prod_{i \in I} X_{i}=\left\{x: x\right.$ is a function of $I$ with $x_{i} \in X_{i}$ for each $\left.i \in I\right\}$.

(8) $\mu$ is a Carathéodory measure on $X$ if and only if $\mu$ is a function on the family of all subsets of $X$ such that $\mu(\varnothing)=0$ and $0 \leqq \mu(A) \leqq \sum_{n \in \omega} \mu\left(B_{n}\right) \leqq \infty$ whenever $A \subset \mathbf{U}_{n \in \omega} B_{n} \subset X$.

(9) For $\mu$ a Carathéodory measure on $X, A$ is $\mu$-measurable if and only if $A \subset X$ and for every $B \subset X, \mu(B)=\mu(B \cap A)+\mu(B-A)$. $\mathscr{K}_{\mu}=\{A: A$ is $\mu$-measurable $\}$.

(10) $\mu$ is a $\mathscr{G}$-outer measure on $X$ if and only if $\mu$ is a Carathéodory measure on $X, \mathscr{G} \subset \mathscr{L}_{\mu}$, and for every $A \subset X, \mu(A)=\inf \{\mu(B): B \in \mathscr{G}$ and $A \subset B$.

(11) $\mu$ is the Carathéodory measure on $X$ generated by $\tau$ and $\mathscr{G}$ if and only if $\mathscr{G}$ is a family of subsets of $X, \tau(A) \geqq 0$ for every $A \in \mathscr{G}$, and for $B \subset X \mu(B)=\inf \left\{\sum_{A \in \mathscr{H}} \tau(A): \mathscr{H} \subset \mathscr{G}, \mathscr{H}\right.$ is countable and $B \subset \mathbf{U}_{A \in \mathscr{C}} A$ \}. 
(12) For $X$ a topological space, $\mu$ is a Radón outer measure on $X$ if and only if $\mu$ is a Carathéodory measure on $X$ such that

(1) open sets are $\mu$-measurable,

(2) If $C$ is compact then $\mu(C)<\infty$,

(3) if $A$ is open then $\mu(A)=\sup \{\mu(C): C$ is compact, $C \subset A\}$,

(4) if $B \subset X$ then $\mu(B)=\inf \{\mu(A): A$ is open, $B \subset A\}$.

(13) For $X$ a topological space, $\mu$ is the topological measure cranked by $\tau$ if and only if $\tau$ is a function on the family of closed compact subsets of $X, \tau_{*}(A)=\sup \{\tau(C): C$ is closed compact and $C \subset A\}$ for $A$ an open subset of $X$, and $\mu$ is the Carathéodory measure on $X$ generated by $\tau_{*}$ and the family of open subsets of $X$.

(14) RemaRks. We mention here two well known facts about Carathéodory measures:

(1) The Carathéodory measure on $X$ generated by $\tau$ and $\mathscr{G}$ is in fact a Carathéodory measure on $X$.

(2) If $X$ is locally compact and regular and $\tau$ is a function on the family of closed compact subsets of $X$ such that for $A, B$ closed and compact we have $0 \leqq \tau(A) \leqq \tau(A \cup B) \leqq \tau(A)+\tau(B)<\infty$ and $\tau(A \cup B)=\tau(A)+\tau(B)$ if $A \cap B=\varnothing$, then the topological measure cranked by $\tau$ is a Radón outer measure on $X$. (See for example Sion [8].)

2. The family $\mathscr{G}$ of cylinders. Throughout this paper we suppose that $T$ is any index set and that for each $t \in T, Y_{t}$ is a locally compact, $\sigma$-compact and regular topological space.

\subsection{Definitions.}

(1) $X=\prod_{t \in T} Y_{t}$.

(2) $I$ is the set of nonvoid finite subsets of $T$, ordered by inclusion.

For $i, j \in I$ with $i \subset j$

(3) $X_{i}=\Pi_{t \in i} Y_{t}$ is equipped with the product topology (which is locally compact, $\sigma$-compact and regular),

(4) $\mathscr{K}_{i}$ is the family of closed compact subsets of $X_{i}$,

(5) $\pi_{i}$ (respectively $\pi_{i j}$ ) is the canonical projection of $X$ (respectively $X_{j}$ ) onto $X_{i}$,

(6) For $A \subset X_{i}$, cyl $A=\pi_{i}^{-1}[A]$.

If no confusion is possible we will for $t \in T$ identify $t$ and $\{t\}, Y_{t}$ and $X_{\{t\}}$. Thus $Y_{t}=X_{\{t\}}=X_{t}$ and $\mathscr{K}_{\{t\}}=\mathscr{K}_{t}$.

\subsection{Definitions.}

(1) $\mathscr{C}=\left\{\alpha\right.$ : there exists $i \in I$ and $\beta \in \mathscr{K}_{i}$ with $\left.\alpha=\operatorname{cyl} \beta\right\}$. Thus $\mathscr{C}$ is the family of cylinder sets which for some $i \in I$ have a compact base in $X_{i}$. 
(2) $\mathscr{G}_{0}$ is the closure under finite intersections of the family of complements of sets in $\mathscr{C}$.

(3) $\mathscr{G}$ is the closure of $\mathscr{G}_{0}$ under countable unions.

The essential properties of $\mathscr{C}$ are the following:

\section{THEOREM 2.3. $\mathscr{C}$ is a compact family.}

COROLlaRY 2.4. The closure of $\mathscr{C}$ under finite unions is a compact family.

CoRollary 2.5. If $\alpha \in \mathscr{C}$ and, for each $n \in \omega, B_{n} \in \mathscr{G}_{0}$ with $\alpha \subset \bigcup_{n \in \omega} B_{n}$ then there exists $N \in \omega$ such that $\alpha \subset \bigcup_{n=0}^{N} B_{n}$.

Proof of 2.3. Let $\mathscr{A}$ be any subfamily of $\mathscr{C}$ such that for any nonvoid finite $B \subset \mathscr{A}$ we have $\bigcap_{\alpha \in B} \alpha \neq \varnothing$. For each $\alpha \in \mathscr{A}$ let $i_{\alpha} \in I$ be such that $\alpha=\operatorname{cyl} \beta$ for some $\beta \in \mathscr{K}_{i_{\alpha}}$. Let $S=\bigcup_{\alpha \in \mathscr{A}} i_{\alpha}$ and for each $t \in S$ choose $\alpha_{t} \in \mathscr{A}$ with $t \in i_{\alpha_{t}}$ and let $C_{t}=\pi_{t}\left[\alpha_{t}\right]$. Then $C_{t}$ is compact in $X_{t}$ and $C_{t} \neq \varnothing$. Let $z$ be a fixed point in $X$ with $z_{t} \in C_{t}$ for each $t \in S$.

Then $C=\left\{x \in X: x_{t} \in C_{t}\right.$ for $t \in S$ and $x_{t}=z_{t}$ for $\left.t \in T-S\right\}$ is a compact subset of $X$ with respect to the product topology. Now let $\mathscr{B}$ be the family of nonvoid finite subsets of $\mathscr{A}$. Then $\mathscr{B}$ is directed by inclusion. If for each $B \in \mathscr{B}$ we let $T_{B}=\mathbf{U}_{\alpha \in B} i_{\alpha}$, then $T_{B}$ is finite. For each $B \in \mathscr{B}$ choose $y^{B} \in \bigcap_{\beta \in B} \beta \cap \bigcap_{t \in T_{B}} \alpha_{t}$. Then $y_{t}^{B} \in C_{t}$ for each $t \in T_{B}$ and if $x^{B}$ is defined by $x_{t}^{B}=y_{t}^{B}$ for $t \in T_{B}$ and $x_{t}^{B}=z_{t}$ for $t \in T-T_{B}$, then $x^{B} \in \bigcap_{\beta \in B} \beta$ and $x^{B} \in C$. Hence $\left\{x^{B} ; B \in \mathscr{B}\right\}$ is a net in $C$, and since $C$ is compact, this net has a cluster point $x$. If $\alpha \in \mathscr{A}$ then $x^{B} \in \alpha$ for any $B \in \mathscr{B}$ with $\{\alpha\} \subset B$. Therefore the net $\left\{x^{B} ; B \in \mathscr{B}\right\}$ is eventually in $\alpha$ for each $\alpha \in \mathscr{A}$. Hence $x \in \alpha$ for each $\alpha \in \mathscr{A}$ and so $\bigcap_{\alpha \in \mathscr{A}} \alpha \neq \varnothing$.

Proof of 2.4. See Meyer [5] p. 33.

Proof of 2.5. Immediate from the definition of $\mathscr{G}_{0}$ and 2.4 .

The following well known elementary lemma will be needed later:

Lemma 2.6. If $i \in I, A, B$ are open in $X_{i}, \gamma \in \mathscr{K}_{i}$ and $\gamma \subset A \cup B$, then there exist $\alpha, \beta \in \mathscr{K}_{i}$ with $\alpha \subset A, \beta \subset B$ and $\alpha \cup \beta=\gamma$.

3. The family $M$ of measures.

3.1. Definition. $\boldsymbol{M}=\{\mu: \mu$ is a bounded outer measure on $X$ such that 
(1) $\mathscr{G} \subset \mathscr{K}_{\mu}$,

(2) $\mu(A)=\sup \{\mu(\alpha): \alpha \in \mathscr{C}$ and $\alpha \subset A\}$ for $A \in \mathscr{C}$,

(3) $\mu(B)=\inf \{\mu(A): A \in \mathscr{G}$ and $B \subset A\}$ for $B \subset X\}$.

3.2. Definitions. For any set function $\tau$ on $\mathscr{C}$

(1) $\tau$ satisfies condition (a) if and only if $\tau$ is bounded, $\tau(\varnothing)=0$ and for every $i \in I$ and $\alpha, \beta \in \mathscr{K}_{i} 0 \leqq \tau(\operatorname{cyl} \alpha) \leqq \tau(\operatorname{cyl} \alpha \cap \operatorname{cyl} \beta) \leqq \tau(\operatorname{cyl} \alpha)+$ $\tau(\operatorname{cyl} \beta)$ and $\tau(\operatorname{cyl} \alpha \cup \operatorname{cyl} \beta)=\tau(\operatorname{cyl} \alpha)+\tau(\operatorname{cyl} \beta)$ if $\alpha \cap \beta=\varnothing$.

(2) $\tau$ satisfies condition (b) if and only if for every $i \in I, \alpha \in \mathscr{K}_{i}$, $t \in T-i$ and sequence $C$ in $\mathscr{K}_{t}$ with $C_{n} \subset$ interior $C_{n+1}$ for $n \in \omega$ and $C_{n} \uparrow X_{t}$, if $j=i \cup\{t\}$ and $\beta_{n}=\left\{x \in X_{j}: x \mid i \in \alpha\right.$ and $\left.x_{t} \in C_{n}\right\}$ then $\tau\left(\operatorname{cyl} \beta_{n}\right) \uparrow \tau(\operatorname{cyl} \alpha)$. (Note that we certainly have cyl $\left.\beta_{n} \uparrow \operatorname{cyl} \alpha.\right)$

(3) $\tau_{*}(A)=\sup \{\tau(\alpha): \alpha \in \mathscr{C}$ and $\alpha \subset A\}$ for $A \in \mathscr{G}$.

The key results of this section are summed up in the following

THEOREM 3.3. Let $\tau$ satisfy conditions (a) and (b) and $\mu$ be the Carathéodory measure on $X$ generated by $\tau_{*}$ and $\mathscr{G}$. Then

(1) $\mu \in M$ and $\mu$ agrees with $\tau_{*}$ on $\mathscr{G}$,

(2) if $i \in I, \mu_{i}(A)=\mu(\operatorname{cyl} A)$ for $A \subset X_{i}, \tau_{i}(\alpha)=\tau(\operatorname{cyl} \alpha)$ for $\alpha \in \mathscr{K}_{i}$ and $\nu_{i}$ is the topological outer measure on $X_{i}$ cranked by $\tau_{i}$, then $\nu_{i}$ is a bounded Radón outer measure and $\mu_{i}$ agrees with $\nu_{i}$ on $\mathscr{C l}_{\nu_{\imath}}$.

For the proof of this theorem two preliminary lemmas are needed.

Lemma A. Let $\tau$ satisfy condition (b). Then for $i, j \in I$ with $i \subset j$ and $\alpha \in \mathscr{K}_{i}$ we have $\tau(\operatorname{cyl} \alpha)=\sup \left\{\tau(\operatorname{cyl} \beta): \beta \in \mathscr{K}_{j}\right.$ and $\left.\operatorname{cyl} \beta \subset \operatorname{cyl} \alpha\right\}$.

Proof. Follows easily from condition (b) and induction.

LEMMA B. If $\tau$ satisfies conditions (a) and (b) then $\tau_{*}$ is countably subadditive on $\mathscr{G}$.

Proof. Let $A_{n} \in \mathscr{G}$ for $n \in \omega, \varepsilon>0$ and $\alpha \in \mathscr{C}$ with $\alpha \subset \bigcup_{n \in \omega} A_{n}$. For each $n \in \omega, A_{n}=\mathbf{U}_{m \in \omega} B_{n m}$ where $B_{n m} \in \mathscr{G}_{0}$. So $\alpha \subset \mathbf{U}_{n \in \omega} \mathbf{U}_{m \in \omega} B_{n m}$ and hence by Corollary 2.5 there exist $N, M \in \omega$ such that $\alpha \subset \bigcup_{n=0}^{N} \bigcup_{m=0}^{M} B_{n m}$. Let, for $0 \leqq n \leqq N, E_{n}=\bigcup_{m=0}^{M} B_{n m}$ and $i_{n}$ be such that $E_{n}=\operatorname{cyl} A_{n}$ for some $A_{n} \subset X_{i_{n}}$. Let $i_{\alpha} \in I$ be such that $\alpha=\operatorname{cyl} \gamma$ for some $\gamma \in \mathscr{K}_{i_{\alpha}}$. Let $i=i_{\alpha}, \mathbf{U}_{n=0}^{N}, i_{n}$, then $i$ is finite and $i_{\alpha} \subset i$. By Lemma $\mathrm{A}$ of this section choose $\beta \in \mathscr{K}_{i}$ with $\operatorname{cyl} \beta \subset \alpha$ and $\tau(\alpha) \leqq \tau(\operatorname{cyl} \beta)+\varepsilon$. Now for $0 \leqq n \leqq N, \pi_{i}\left[E_{n}\right]$ is open in $X_{i}$ and $\beta \subset \pi_{i}[\alpha] \subset \bigcup_{n=0}^{N} \pi_{i}\left[E_{n}\right]$. 
By Lemma 2.6 and induction we can, for $0 \leqq n \leqq N$, find $\beta_{n} \in \mathscr{K}_{i}$ with $\beta_{n} \subset \pi_{i}\left[E_{n}\right]$ and $\beta=\bigcup_{n=0}^{N} \beta_{n}$. Then cyl $\beta=\bigcup_{n=0}^{N} \operatorname{cyl} \beta_{n} \subset \bigcup_{n=0}^{N} E_{n}$. By condition (a) we have $\tau(\operatorname{cyl} \beta) \leqq \sum_{n=0}^{N} \tau\left(\operatorname{cyl} \beta_{n}\right)$. Hence $\tau(\alpha) \leqq$ $\tau(\operatorname{cyl} \beta)+\varepsilon \leqq \sum_{n=0}^{N} \tau\left(\operatorname{cyl} \beta_{n}\right)+\varepsilon \leqq \sum_{n=0}^{N} \tau_{*}\left(E_{n}\right)+\varepsilon \leqq \sum_{n \in \omega} \tau_{*}\left(A_{n}\right)+\varepsilon$. It follows that $\tau_{*}\left(\bigcup_{n \in \omega} A_{n}\right)=\sup \left\{\tau(\alpha): \alpha \in \mathscr{C}\right.$ and $\left.\alpha \subset \bigcup_{n \in \omega} A_{n}\right\} \leqq \sum_{n \in \omega} \tau_{*}\left(A_{n}\right)$. We now proceed to prove Theorem 3.3.

Proof of 3.3(1) Since, by Lemma B, $\tau_{*}$ is countably subadditive on $\mathscr{G}$ and since $\mathscr{C}$ is closed under countable unions we have for $A \in \mathscr{G}, \mu(A)=\tau_{*}(A)$ and therefore for $B \subset X, \mu(B)=\inf \{\mu(A): A \in \mathscr{G}$ and $B \subset A\}$. Furthermore, since clearly $\mu(\alpha) \geqq \tau(\alpha)$ for each $\alpha \in \mathscr{C}$, it follows that for $A \in \mathscr{C}, \mu(A)=\sup \{\mu(\alpha): \alpha \in \mathscr{C}$ and $\alpha \subset A\}$. Now, since $\mu$ certainly is a bounded Carathéodory measure on $X$, all that remains is to show $\mathscr{C} \subset \mathscr{C l}_{\mu}$. So let $A \in \mathscr{C}, B \subset X$ and $\varepsilon>0$. Choose $B^{\prime} \in \mathscr{C}$ with $B \subset B^{\prime}$ and $\mu\left(B^{\prime}\right) \leqq \mu(B)+\varepsilon$. Let $\alpha \in \mathscr{C}$ with $\alpha \subset B^{\prime} \cap A$ and $\mu\left(B^{\prime} \cap A\right) \leqq \mu(\alpha)+\varepsilon$. Let $\beta \in \mathscr{C}$ with $\beta \subset B^{\prime}-\alpha$ and $\mu\left(B^{\prime}-\alpha\right) \leqq$ $\mu(\beta)+\varepsilon$. By Lemma A we can suppose that $\alpha \cup \beta \in \mathscr{C}$ also. Then $\mu(B \cap A)+\mu(B-A) \leqq \mu\left(B^{\prime} \cap A\right)+\mu\left(B^{\prime}-\alpha\right) \leqq \mu(\alpha)+\mu(\beta)+2 \varepsilon=$ $\mu(\alpha \cup \beta)+2 \varepsilon \leqq \mu\left(B^{\prime}\right)+2 \varepsilon \leqq \mu(B)+3 \varepsilon$.

Hence $\mu(B \cap A)+\mu(B-A)=\mu(B)$ for all $B \subset X$. It follows that $\mathscr{S} \subset \mathscr{M}$.

Proof of 3.3(2) Let $\nu_{i}$ be the topological outer measure on $X_{i}$ cranked by $\tau_{i}$. By condition (a) and Remark 1.14.2 we have that $\nu_{i}$ is a bounded Radón outer measure on $X_{i}$. Let $A$ be open in $X_{i}$. Since $X_{i}-A$ is closed and $X_{i}$ is $\sigma$-compact, we can for $n \in \omega$ choose $C_{n} \in \mathscr{K}_{i}$ such that $C_{n} \uparrow\left(X_{i}-A\right)$. Then $A=\bigcap_{n \in \omega}\left(X_{i}-C_{n}\right)$. Since $\operatorname{cyl}\left(X_{i}-C_{n}\right) \in$ we have

$$
\begin{aligned}
\mu_{i}\left(X_{i}-C_{n}\right) & =\mu\left(\operatorname{cyl}\left(X_{i}-C_{n}\right)\right)=\tau_{*}\left(\operatorname{cyl}\left(X_{i}-C_{n}\right)\right) \\
& =\sup \left\{\tau(\beta): \beta \in \mathscr{C} \text { and } \beta \subset \operatorname{cyl}\left(X_{i}-C_{n}\right)\right\} \\
& =\sup \left\{\tau(\operatorname{cyl} \alpha): \alpha \in \mathscr{X}_{i} \text { and } \alpha \subset\left(X_{i}-C_{n}\right)\right\} \\
& =\sup \left\{\tau_{i}(\alpha): \alpha \in \mathscr{K}_{i} \text { and } \alpha \subset\left(X_{i}-C_{n}\right)\right\} \\
& =\nu_{i}\left(X_{i}-C_{n}\right) .
\end{aligned}
$$

Furthermore since the $X_{i}-C_{n}$ are $\mu_{i}$-measurable as well as $\nu_{i}$-measurable we have $\mu_{i}(A)=\lim _{n \in \omega} \mu_{i}\left(X_{i}-C_{n}\right)=\lim _{n \in \omega} \nu_{i}\left(X_{i}-C_{n}\right)=\nu_{i}(A)$. Hence $\mu_{i}$ and $\nu_{i}$ agree on open sets. If $D \subset X_{i}$ then $\nu_{i}(D)=\inf \left\{\nu_{i}(A): A\right.$ is open in $X_{i}$ and $\left.D \subset A\right\}=\inf \left\{\mu_{i}(A): A\right.$ is open in $X_{i}$ and $\left.D \subset A\right\} \geqq \mu_{i}(D)$. Hence $\mu_{i} \leqq \nu_{i}$ always.

Now let $B \in \mathscr{A}_{\nu}$. Given $\varepsilon>0$ choose $A$ open in $X_{i}$ with $B \subset A$ and $\nu_{i}(A)<\nu_{i}(B)+\varepsilon$. Since $\nu_{i}(A)=\nu_{i}(B)+\nu_{i}(A-B)$ we have $\nu_{i}(A-B)<\varepsilon$ and consequently $\mu_{i}(A-B)<\varepsilon$. But $\mu_{i}(A) \leqq \mu_{i}(A-B)+$ 
$\mu_{i}(B)<\mu_{i}(B)+\varepsilon$. Hence

$$
\begin{aligned}
\mu_{i}(B) & =\inf \left\{\mu_{i}(A): A \text { is open in } X_{i} \text { and } B \subset A\right\} \\
& =\inf \left\{\nu_{i}(A): A \text { is open in } X_{i} \text { and } B \subset A\right\} \\
& =\nu_{i}(B) .
\end{aligned}
$$

3.4. $M$ as related to projective limit measures. Suppose that for each $i \in I, \mathscr{B}_{i}$ is the $\sigma$-ring generated by $\mathscr{K}_{i}$ and $\nu_{i}$ is a measure on $\mathscr{B}_{i}$. We call $\left\{\nu_{i}: i \in I\right\}$ a projective system of measures if whenever $i, j \in I$ with $i \subset j$ we have for $A \in \mathscr{B}_{i}$

$$
\nu_{i}(A)=\nu_{\jmath}\left(\pi_{i j}^{-1}[A]\right) .
$$

We say that the projective system $\left\{\nu_{i}: i \in I\right\}$ admits a projective limit measure $\nu$ if $\nu$ is a measure on the $\sigma$-ring $\mathscr{B}$ of subsets of $X$ generated by $\left\{\right.$ cyl $B: B \in \mathscr{B}_{i}$ for some $\left.i \in I\right\}$ such that for each $i \in I$ and $A \in \mathscr{S}_{i}$, $\nu(\operatorname{cyl} A)=\nu_{i}(A)$. Such a measure $\nu$, if it exists, is unique and can thus be called the projective limit measure of the system $\left\{\nu_{i}: i \in I\right\}$.

For more general definitions of projective or inverse systems of measures see Choksi [1], Mallory [4] or Meyer [5].

Now, if for $i \in I$ we call $\nu_{i}$ a bounded regular Borel measure whenever $\nu_{i}$ is a bounded measure on $\mathscr{B}_{i}$ such that for every $A \in \mathscr{S}_{i}$

$$
\begin{aligned}
\nu_{i}(A) & =\inf \left\{\nu_{i}(B): B \text { is open and } A \subset B\right\} \\
& =\sup \left\{\nu_{i}(C): C \in \mathscr{K}_{i} \text { and } C \subset A\right\}
\end{aligned}
$$

we then have

THEOREM 3.4.1. $\mu \in M$ if and only if $\mu$ is a Couter measure on $X$ and $\mu \mid \mathscr{B}$ is the projective limit measure of a projective system $\left\{\mu_{i}: i \in I\right\}$ of bounded regular Borel measures $\mu_{i}$ on $\mathscr{B}_{i}$.

Proof. Suppose $\mu \in M$. Then $\mu$ is a $\mathscr{C}$-outer measure on $X$. If $\mu_{i}(A)=\mu(\operatorname{cyl} A)$ for $A \in \mathscr{\mathscr { S }}_{i}$ then clearly $\left\{\mu_{i}: i \in I\right\}$ forms a projective system of measures and $\mu \mid \mathscr{B}$ is clearly the projective limit measure of this system. Using 3.3(2) one can easily check that each $\mu_{i}$ is in fact a bounded regular Borel measure on $\mathscr{B}_{i}$.

Conversely let $\mu$ be a $\mathscr{S}$-outer measure on $X$ and $\mu \mid \mathscr{B}$ be the projective limit measure of a projective system $\left\{\mu_{i}: i \in I\right\}$ of bounded regular Borel measures $\mu_{i}$ on $\mathscr{B}_{i}$. Let for each $i \in I$ and $\alpha \in \mathscr{K}_{i}$, $\tau(\operatorname{cyl} \alpha)=\mu_{i}(\alpha)$. Then $\tau$ is a set function on $\mathscr{C}$ satisfying conditions (a) and (b). Let $\nu$ be the Carathéodory measure on $X$ generated by $\tau_{*}$ and $\mathscr{C}$. Then by 3.3(1), $\nu \in M$. Clearly $\nu \mid \mathscr{B}$ is the projective limit measure of the system $\left\{\nu_{i}: i \in I\right\}$ where $\nu_{i}(A)=\nu(\operatorname{cyl} A)$ for $A \in \mathscr{B}_{i}$. From 3.3(2), we see that $\mu_{i}=\nu_{i}$ for each $i \in I$. Hence $\mu|\mathscr{B}=\nu| \mathscr{B}$. 
Since $\mathscr{G} \subset \mathscr{B}, \mu|\mathscr{G}=\nu| \mathscr{G}$ and therefore, since both $\mu$ and $\nu$ are $\mathscr{G}$-outer measures on $X$, we have $\mu=\nu$. Hence $\mu \in \boldsymbol{M}$.

4. The representation theorem.

4.1. Definitions. (1) For $i \in I, C_{0}\left(X_{i}\right)$ is the set of continuous real valued functions on $X_{i}$ with compact support.

(2) For $i \in I$ and $h \in C_{0}\left(X_{i}\right)$, cyl $h$ is the function on $X$ given by $(\operatorname{cyl} h)(x)=h(x \mid i)$ for every $x \in X$.

(3) $\boldsymbol{F}=\left\{f\right.$ : there exists $i \in I$ and $h \in C_{0}\left(X_{i}\right)$ with $\left.f=\operatorname{cyl} h\right\}$.

4.2. Definitions. (1) $L=(l: l$ is a positive linear functional on the linear span of $\boldsymbol{F}$ such that

(1) there exists $K>0$ with $|l(f)| \leqq K\|f\|_{\infty}$ for all $f \in \boldsymbol{F}$,

(2) if $i, j \in I$ with $i \subset j, f \in C_{0}\left(X_{i}\right)$ and, for $n \in \omega, f_{n} \in C_{0}\left(X_{j}\right)$ with $\operatorname{cyl} f_{n} \uparrow \operatorname{cyl} f$ then $l\left(\operatorname{cyl} f_{n}\right) \uparrow l(\operatorname{cyl} f)$. $\}$

(Note that in the definition of $\boldsymbol{L}$ above, condition (1) does not necessarily imply condition (2).)

(2) For $l \in \boldsymbol{L}, \tau^{l}$ is the set function on $\mathscr{G}$ given by $\tau^{l}(\alpha)=$ $\inf \left\{l(f): 1_{\alpha} \leqq f \in \boldsymbol{F}\right\}$ for $\alpha \in \mathscr{G}$.

Our basic theorem now is

THEOREM 4.3. For each $l \in \boldsymbol{L}$ there exists a unique $\mu^{l} \in \boldsymbol{M}$ such that the relationship $l(f)=\int f d \mu^{l}$ holds for all $f \in F$. Moreover the mapping $l \rightarrow \mu^{l}$ is an isomorphism between $\boldsymbol{L}$ and $\boldsymbol{M}$.

For the proof of this theorem we will need three preliminary lemmas.

Lemma C. For $l \in L, i \in I$ and $\alpha \in \mathscr{K}_{i}$,

$$
\tau^{l}(\operatorname{cyl} \alpha)=\inf \left\{l(\operatorname{cyl} f): 1_{\alpha} \leqq f \in C_{0}\left(X_{i}\right)\right\} \text {. }
$$

Proof. Suppose $h \in \boldsymbol{F}$ and $1_{\text {cyl } \alpha} \leqq h$. We want to find $f \in C_{0}\left(X_{i}\right)$ with $1_{\alpha} \leqq f$ and cyl $f \leqq h$. By definition there exists $j \in I$ and $g \in C_{0}\left(X_{j}\right)$ such that $h=\operatorname{cyl} g$. Let $k=i \cup j$. For $z \in X_{k}$ let $h_{k}(z)=h(y)$ for some $y \in X$ with $y \mid k=z$. (Note that $h_{k}(z)$ is independent of $y$ provided $y \mid k=z$, and that cyl $h_{k}=h$.) Since $g \in C_{0}\left(X_{j}\right)$ and $h_{k}(z)=g(z \mid j)$ we have that $h_{k}$ is uniformly continuous on $X_{k}$. Hence if for $x \in X_{i}$

$$
\begin{aligned}
f^{*}(x) & =\inf \left\{h_{k}(z): z \in X_{k} \text { and } z \mid i=x\right\} \\
& =\inf \{h(y): y \in X \text { and } y \mid i=x\}
\end{aligned}
$$


then $f^{*}$ is continuous on $X_{i}$. Moreover it is clear that cyl $f^{*} \leqq h$ and $1_{\alpha} \leqq f^{*}$. Since $X_{i}$ is locally compact and regular there exists $f \in C_{0}\left(X_{i}\right)$ with $1_{\alpha} \leqq f \leqq f^{*}$. Hence $1_{\alpha} \leqq f$ and cyl $f \leqq h$. It follows that

$$
\begin{aligned}
\tau^{l}(\operatorname{cyl} \alpha) & =\inf \left\{l(h): 1_{\text {cyl } \alpha} \leqq h \in \boldsymbol{F}\right\} \\
& =\inf \left\{l(\operatorname{cyl} f): 1_{\alpha} \leqq f \in C_{0}\left(X_{i}\right)\right\}
\end{aligned}
$$

Lemma D. Let $l \in L, i \in I, \alpha \in \mathscr{K}_{i}$. Then for every $\varepsilon>0$ there exists $A$ open in $X_{i}$ with $\alpha \subset A$ such that for any $j \in I$ with $i \subset j$ and $f \in C_{0}\left(X_{j}\right)$ with $\|f\|_{\infty} \leqq 1$ and $\{x: f(x)>0\} \subset \pi_{i j}^{-1}[A-\alpha]$ we have $l(\operatorname{cyl} f) \leqq \varepsilon$.

Proof. By Lemma C choose $h \in C_{0}\left(X_{i}\right)$ with $1_{\alpha} \leqq h$ and $l(\operatorname{cyl} h) \leqq$ $\tau^{l}(\operatorname{cyl} \alpha)+\varepsilon / 2$. Let

$$
A=\{x:(1+\varepsilon / 1+2 l(\operatorname{cyl} h)) h(x)>1\} .
$$

Then $A$ is open and $\alpha \subset A$. Now let $j \in I$ with $i \subset j$. Suppose first that $g \in C_{0}\left(X_{j}\right)$ with $0 \leqq g \leqq 1$ and support $g \subset \pi_{i j}^{-1}[A-\alpha]$. Let $\beta=$ $\pi_{i j}$ [support $g$ ]. Then $\alpha, \beta$ are disjoint compact subsets of $A$ and so let $V, W$ be disjoint neighborhoods of $\alpha$ and $\beta$ respectively with $V \cup W \subset A$. Let $v, w \in C_{0}\left(X_{i}\right)$ with $1_{\alpha} \leqq v \leqq 1_{U}$ and $1_{\beta} \leqq w \leqq 1_{W}$. Then $v+w \leqq$ $(1+\varepsilon / 1+2 l(\operatorname{cyl} h)) h$ and therefore

$$
\begin{array}{r}
l(\operatorname{cyl} v)+l(\operatorname{cyl} w) \leqq l(\operatorname{cyl} h)+\varepsilon / 2 \\
\leqq \tau^{l}(\operatorname{cyl} \alpha)+\varepsilon \leqq l(\operatorname{cyl} v)+\varepsilon .
\end{array}
$$

Hence $l(\operatorname{cyl} w) \leqq \varepsilon$ and since cyl $g \leqq \operatorname{cyl} w$ we have by condition (2) of $4.2(1)$, that $l(\operatorname{cyl} g) \leqq l(\operatorname{cyl} w)$. Thus $l(\operatorname{cyl} g) \leqq \varepsilon$.

Now let $f \in C_{0}\left(X_{j}\right)$ with $\|f\|_{\infty} \leqq 1$ and $\{x: f(x)>0\} \subset \pi_{i j}^{-1}[A-\alpha]$. For $n \in \omega$ let $\beta_{n}=\{x: f(x) \geqq 1 / n\}$. Then $\beta_{n} \in \mathscr{K}_{j}$ and $\beta_{n} \subset$ interior $\beta_{n+1}$. Let $g_{n} \in C_{0}\left(X_{j}\right)$ with $1_{\beta_{n}} \leqq g_{n} \leqq 1_{\beta_{n+1}}$ and let $f_{n}=f \cdot g_{n}$. Then support $f_{n} \subset \beta_{n+1} \subset \pi_{i j}^{-1}[A-\alpha], 0 \leqq f_{n} \leqq 1$, and hence by the above argument, $l\left(\operatorname{cyl} f_{n}\right) \leqq \varepsilon$. Since $f_{n} \uparrow f^{+}$we have by condition (2) of 4.2(1), that $l\left(\operatorname{cyl} f_{n}\right) \uparrow l\left(\operatorname{cyl} f^{+}\right)$. It follows that $l\left(\operatorname{cyl} f^{+}\right) \leqq \varepsilon$ and therefore $l(\operatorname{cyl} f) \leqq \varepsilon$.

Lemma E. For $l \in \boldsymbol{L}, \tau^{l}$ satisfies conditions (a) and (b).

Proof. Condition (a) follows easily from Lemma C using well known standard arguments. To prove condition (b), let $i \in I, \alpha \in \mathscr{K}_{i}$, $t \in T-i$ and $C$ be a sequence in $\mathscr{K}_{t}$ with $C_{n} \subset$ interior $C_{n+1}$ for $n \in \omega$, and $C_{n} \uparrow X_{t}$. Let $j=i \cup\{t\}$ and $\beta_{n}=\left\{x \in X_{j}: x \mid i \in \alpha\right.$ and $\left.x_{t} \in C_{n}\right\}$. Then cyl $\beta_{n} \uparrow$ cyl $\alpha$. Given $\varepsilon>0$, by Lemma $\mathrm{D}$, there exists $A$ open in 
$X_{i}$ with $\alpha \subset A$ such that for any $g \in C_{0}\left(X_{j}\right)$ with $\|g\|_{\infty} \leqq 1$ and $\{x: g(x)>0\} \subset \pi_{i j}^{-1}[A-\alpha]$ we have $l(\operatorname{cyl} g) \leqq \varepsilon$. Choose $f \in C_{0}\left(X_{i}\right)$ with $1_{\alpha} \leqq f \leqq 1_{A}$ and let $k_{n} \in C_{0}\left(X_{t}\right)$ with $1_{C_{n}} \leqq k_{n} \leqq 1_{C_{n+1}}$.

For $x \in X_{j}$ let $f_{n}(x)=f(x \mid i) \cdot k_{n}\left(x_{t}\right)$. Then $f_{n} \in C_{0}\left(X_{j}\right), 1_{\beta_{n}} \leqq f_{n}$ and cyl $f_{n} \uparrow$ cyl $f$. By Lemma $\mathrm{C}$ choose $h_{n} \in C_{0}\left(X_{j}\right)$ with $1_{\beta_{n}} \leqq h_{n} \leqq f_{n}$ and $l\left(\operatorname{cyl} h_{n}\right) \leqq \tau^{l}\left(\operatorname{cyl} \beta_{n}\right)+\varepsilon$. We note that $f_{n}-h_{n+1} \in C_{0}\left(X_{j}\right),\left\|f_{n}-h_{n+1}\right\|_{\infty} \leqq 1$ and $\left\{x:\left(f_{n}-h_{n+1}\right)(x)>0\right\} \subset \pi_{i j}^{-1}[A-\alpha]$. Hence by Lemma $\mathrm{D}$,

$$
l\left(\operatorname{cyl}\left(f_{n}-h_{n+1}\right)\right)<\varepsilon \text {. }
$$

Since $f_{n}=f_{n}-h_{n+1}+h_{n+1}$ we have $l\left(\operatorname{cyl} f_{n}\right)=l\left(\operatorname{cyl}\left(f_{n}-h_{n+1}\right)\right)+l\left(\operatorname{cyl} h_{n+1}\right)$

$$
\leqq l\left(\operatorname{cyl} h_{n+1}\right)+\varepsilon \leqq \tau^{l}\left(\operatorname{cyl} \beta_{n+1}\right)+2 \varepsilon .
$$

Hence

$$
\begin{aligned}
& \tau^{l}(\operatorname{cyl} \alpha) \leqq l(\operatorname{cyl} f)=\lim _{n \in \omega} l\left(\operatorname{cyl} f_{n}\right) \\
& \quad \leqq \lim _{n \in \omega} l\left(\operatorname{cyl} h_{n+1}\right)+\varepsilon \leqq \lim _{n \in \omega} \tau^{l}\left(\operatorname{cyl} \beta_{n+1}\right)+2 \varepsilon .
\end{aligned}
$$

Thus $\tau^{l}(\operatorname{cyl} \alpha) \leqq \lim _{n \in \omega} \tau^{l}\left(\operatorname{cyl} \beta_{n}\right)$ and since certainly the reverse inequality holds, we have $\tau^{l}(\operatorname{cyl} \alpha)=\lim _{n \in \omega} \tau^{l}\left(\operatorname{cyl} \beta_{n}\right)$.

Proof of 4.3. Let $l \in \boldsymbol{L}$. By Lemma E, $\tau^{l}$ satisfies conditions (a) and (b) and hence by 3.3.1 the Carathéodory outer measure $\mu^{l}$ on $X$ generated by $\tau_{*}^{l}$ and $\mathscr{G}$ is in $\boldsymbol{M}$.

Now suppose $f \in \boldsymbol{F}$. By definition there exists $i \in I$ and $h \in C_{0}\left(X_{i}\right)$ such that $f=\operatorname{cyl} h$. If for every $A \subset X_{i}$ we let $\mu_{i}^{l}(A)=\mu^{l}(\operatorname{cyl} A)$ then $\int \operatorname{cyl} h d \mu^{l}=\int h d \mu_{i}^{l}$. If for $\alpha \in \mathscr{K}_{i}$ we let $\tau_{i}^{l}(\alpha)=\tau^{l}(\operatorname{cyl} \alpha)$ and let $\nu_{i}^{l}$ be the topological outer measure on $X_{i}$ cranked by $\tau_{i}^{l}$, then by $3.3(2), \nu_{i}^{l}$ is a Radón outer measure on $X_{i}$ and $\mu_{i}^{l}$ agrees with $\nu_{i}^{l}$ on all $\nu_{i}^{l}$-measurable sets. Hence since $h \in C_{0}\left(X_{i}\right)$ we have

$$
\int h d \nu_{i}^{l}=\int h d \mu_{i}^{l}
$$

Furthermore if $l_{i}(g)=l(\operatorname{cyl} g)$ for $g \in C_{0}\left(X_{i}\right)$ then $l_{i}$ is a positive continuous linear functional on $C_{0}\left(X_{i}\right)$ and by Lemma $\mathrm{C}$

$$
\tau_{i}^{l}(\alpha)=\inf \left\{l_{i}(g): 1_{\alpha} \leqq g \in C_{0}\left(X_{i}\right)\right\} \text { for } \alpha \in \mathscr{K}_{i} \text {. }
$$

Hence by the Riesz Representation Theorem $l_{i}$ and $\nu_{i}^{l}$ satisfy the relationship

$$
\begin{aligned}
l_{i}(g) & =\int g d \nu_{i}^{l} \text { for all } g \in C_{0}\left(X_{i}\right) . \\
\text { Hence } l(f) & =l(\operatorname{cyl} h)=l_{i}(h)=\int h d \nu_{i}^{l}=\int h d \mu_{i}^{l} \\
& =\int \operatorname{cyl} h d \mu^{l}=\int f d \mu^{l} .
\end{aligned}
$$


To show uniqueness, suppose $\mu \in \boldsymbol{M}$ and $l(f)=\int f d \mu$ for all $f \in \boldsymbol{F}$. For each $i \in I$ let $\mu_{i}(A)=\mu(\operatorname{cyl} A)$ for $A \subset X_{i}, \tau_{i}(\alpha)=\mu(\operatorname{cyl} \alpha)$ for $\alpha \in \mathscr{K}_{i}$ and $\nu_{i}$ be the topological outer measure on $X_{i}$ cranked by $\tau_{i}$. By 3.3(2), $\nu_{i}$ is a Radón outer measure on $X_{i}$ and $\mu_{i}$ agrees with $\nu_{i}$ on $\mathscr{L}_{\nu_{i}}$, hence also on $\mathscr{B}_{i}$. Furthermore for all $f \in C_{0}\left(X_{i}\right)$

$$
\int f d \nu_{i}=\int f d \mu_{i}=l(\operatorname{cyl} f)=\int f d \mu_{i}^{l}=\int f d \nu_{i}^{l}
$$

and therefore by the Riesz representation theorem $\nu_{i}=\nu_{i}^{l}$. It follows that $\mu_{i}$ and $\mu_{i}^{l}$ agree on $\mathscr{B}_{i}$. Hence the projective systems $\left\{\mu_{i} \mid \mathscr{B}_{i}: i \in I\right\}$ and $\left\{\mu_{i}^{l} \mid \mathscr{B}_{i}: i \in I\right\}$ are equal and so their respective projective limit measures, which by 3.4.1 are $\mu \mid \mathscr{B}$ and $\mu^{l} \mid \mathscr{S}$, are also equal. Since $\mathscr{C} \subset \mathscr{B}$ we have that $\mu$ and $\mu^{l}$ agree on $\mathscr{C}$ and so $\mu=\mu^{l}$. The mapping $l \rightarrow \mu^{l}$ is now clearly an isomorphism between $\boldsymbol{L}$ and $\boldsymbol{M}$.

5. Example to show that $\sigma$-compactness of the coordinate spaces is needed. Let $\boldsymbol{R}$ have the discrete topology (which is not $\sigma$-compact) and consider $\boldsymbol{R}^{2}$ with the product topology. For $h \in C_{0}(\boldsymbol{R})$ and $x \in \boldsymbol{R}^{2}$, let $\left(\operatorname{cyl}_{1} h\right)(x)=h\left(x_{1}\right)$ and $\left(\operatorname{cyl}_{2} h\right)(x)=h\left(x_{2}\right)$.

Let $\boldsymbol{F}_{0}=C_{0}\left(\boldsymbol{R}^{2}\right)$

$\boldsymbol{F}_{1}=\left\{f: f=\operatorname{cyl}_{1} h\right.$ for some $\left.h \in C_{0}(\boldsymbol{R})\right\}$

$\boldsymbol{F}_{2}=\left\{f: f=\operatorname{cyl}_{2} h\right.$ for some $\left.h \in C_{0}(\boldsymbol{R})\right\}$.

Using the notations of this paper, we let $T=\{1,2\}, Y_{1}=Y_{2}=\boldsymbol{R}$ with the discrete topology and define $X, \mathscr{C}, \mathscr{C}, \boldsymbol{M}, \boldsymbol{F}$ and $\boldsymbol{L}$ as before.

First we note that $\boldsymbol{F}=\boldsymbol{F}_{0} \cup \boldsymbol{F}_{1} \cup \boldsymbol{F}_{2}$ and that since pairwise intersections of $\boldsymbol{F}_{0}, \boldsymbol{F}_{1}$ and $\boldsymbol{F}_{2}$ consist of the zero element only, every $f$ in the linear span of $\boldsymbol{F}$ has a unique representation as $f=f_{0}+f_{1}+f_{2}$ where $f_{n} \in \boldsymbol{F}_{n}$ for $n=0,1,2$. For fixed $z \in \boldsymbol{R}^{2}$ (which equals $X$ ) define $l$ by $l(f)=f_{0}(z)+2 f_{1}(z)+2 f_{2}(z)$ for $f$ in the linear span of $\boldsymbol{F}$. Then $l \in \boldsymbol{L}$ but we shall show that there is no $\mu \in M$ such that $l(f)=\int f d \mu$ for all $f \in \boldsymbol{F}$. Suppose we did find such a $\mu \in \boldsymbol{M}$. Then if $A=\left\{x \in \boldsymbol{R}^{2}: x_{1}=z_{1}\right\}$ we have $1_{A} \in \boldsymbol{F}_{1} \subset \boldsymbol{F}$ and hence

$$
\mu(A)=\int 1_{A} d \mu=l\left(1_{A}\right)=2 \cdot 1_{A}(z)=2 .
$$

We next note that $1_{\{z\}} \in \boldsymbol{F}_{0} \subset \boldsymbol{F}$ and so

$$
\mu(\{z\})=\int 1_{\{z\}} d \mu=l\left(1_{\{z\}}\right)=1_{\{z\}}(z)=1 .
$$

Furthermore since $A,\{z\}$ and $A-\{z\}$ are all $\mu$-measurable we have 


$$
\mu(A-\{z\})=\mu(A)-\mu(\{z\})=2-1=1
$$

On the other hand $A-\{z\} \subset \boldsymbol{R}^{2}-\{z\}$ which is in $\mathscr{G}$. Hence

$$
\begin{aligned}
\mu(A & -\{z\}) \leqq \mu\left(\boldsymbol{R}^{2}-\{z\}\right) \\
& =\sup \left\{\mu(\alpha): \alpha \in \mathscr{C} \text { and } \alpha \subset \boldsymbol{R}^{2}-\{z\}\right\} \\
& =\sup \left\{l\left(1_{\alpha}\right): \alpha \in \mathscr{C} \text { and } \alpha \subset \boldsymbol{R}^{2}=\{z\}\right\}=0
\end{aligned}
$$

since $l\left(1_{\alpha}\right)=0$ for any $\alpha \in \mathscr{C}$ with $z \notin \alpha$. Hence $A-\{z\}$ would have to have measure zero and one simultaneously, which is impossible.

\section{BIBLIOGRAPHY}

1. I. R. Choksi, Inverse limits of measure spaces, Proc. London Math. Soc. 8 (1958), 321-342.

2. L. Gross, Measurable functions on Hilbert space, Trans. Amer. Math. Soc. 105 (1962), 372-390.

3. “Classical Analysis on Hilbert space," in Analysis in function spaces, M.I.T. Press, Cambridge, Massachusetts, 1964.

4. D. I. Mallory, Limits of inverse systems of measures, thesis, Univ. of British Columbia, 1968.

5. P. A. Meyer, Probabilities and potentials, Blaisdell, 1966.

6. I. E. Segal, Tensor algebras over Hilbert spaces, Trans. Amer. Math. Soc. 81 (1956), 106-134.

7. G. E. Silov, On some questions of analysis in Hilbert space, Part I. Functional Analysis Applic. 1 (1967) (English translation).

8. M. Sion, Lecture notes on measure theory, Biennial Seminar of the Canadian Math. Congress, 1965.

Received October 22, 1968. This research was supported in part by the Summer Research Institute of the Canadian Mathematical Congress.

UNIVERSITY OF BRITISH Columbia

VANCOUVER 8, CANADA 


\section{PACIFIC JOURNAL OF MATHEMATICS}

\section{EDITORS}

H. ROYDEN

Stanford University

Stanford, California

Richard Pierce

University of Washington

Seattle, Washington 98105
J. DugundJI

Department of Mathematics

University of Southern California

Los Angeles, California 90007

BASIL GORDON

University of California

Los Angeles, California 90024

\section{ASSOCIATE EDITORS}

E. F. BECKENBACH

B. H. NeUmanN

F. WOLF

K. YOSHIDA

\section{SUPPORTING INSTITUTIONS}

UNIVERSITY OF BRITISH COLUMBIA

CALIFORNIA INSTITUTE OF TECHNOLOGY

UNIVERSITY OF CALIFORNIA

MONTANA STATE UNIVERSITY

UNIVERSITY OF NEVADA

NEW MEXICO STATE UNIVERSITY

OREGON STATE UNIVERSITY

UNIVERSITY OF OREGON

OSAKA UNIVERSITY

UNIVERSITY OF SOUTHERN CALIFORNIA
STANFORD UNIVERSITY

UNIVERSITY OF TOKYO

UNIVERSITY OF UTAH

WASHINGTON STATE UNIVERSITY

UNIVERSITY OF WASHINGTON

$* * \quad * \quad *$
AMERICAN MATHEMATICAL SOCIETY
CHEVRON RESEARCH CORPORATION
TRW SYSTEMS
NAVAL WEAPONS CENTER




\section{Pacific Journal of Mathematics}

Vol. 30, No. $1 \quad$ September, 1969

William Wells Adams, Simultaneous diophantine approximations and cubic irrationals ..................................... 1

Heinz Bauer and Herbert Stanley Bear, Jr., The part metric in convex

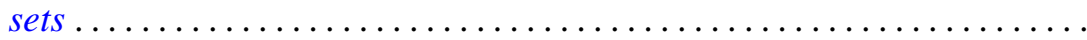

L. Carlitz, A note on exponential sums ...................... 35

Vasily Cateforis, On regular self-injective rings ................. 39

Franz Harpain and Maurice Sion, A representation theorem for measures on infinite dimensional spaces ......................... 47

Richard Earl Hodel, Sum theorems for topological spaces .............. 59

Carl Groos Jockusch, Jr. and Thomas Graham McLaughlin, Countable

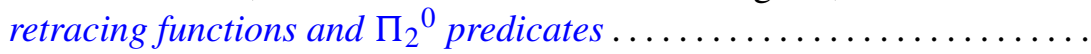

Bjarni Jónsson and George Stephen Monk, Representations of primary

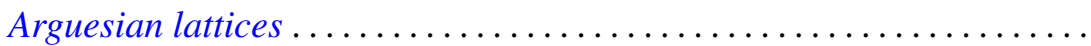

Virginia E. Walsh Knight, A continuous partial order for Peano continua...................................... 141

Kjeld Laursen, Ideal structure in generalized group algebras ........... 155

G. S. Monk, Desargues' law and the representation of primary lattices . . . 175

Hussain Sayid Nur, Singular perturbation of linear partial differential equation with constant coefficients ..........................

Richard Paul Osborne and J. L. Stern, Covering manifolds with cells ... 201

Keith Lowell Phillips and Mitchell Herbert Taibleson, Singular integrals in several variables over a local field...

James Reaves Smith, Local domains with topologically $T$-nilpotent radical....

Donald Platte Squier, Elliptic differential equations with discontinuous coefficients .................................

Tae-il Suh, Algebras formed by the Zorn vector matrix...

Earl J. Taft, Ideals in admissible algebras . .................... 259

Jun Tomiyama, On the tensor products of von Neumann algebras........ 263

David Bertram Wales, Uniqueness of the graph of a rank three group ..... 271

Charles Robert Warner and Robert James Whitley, A characterization of regular maximal ideals ......................... 